
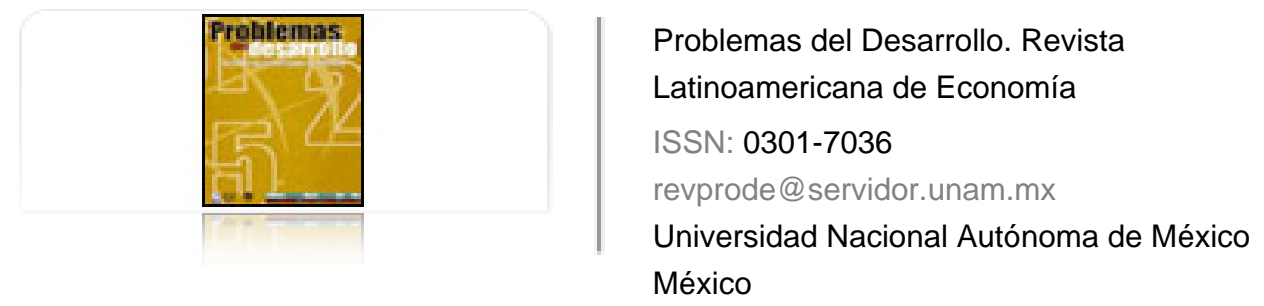

Fraga Castillo, Carlos Alberto; Moreno-Brid, Juan Carlos

Exportaciones, términos de intercambio y crecimiento económico de Brasil y México, de 1960 a 2002: un análisis comparativo

Problemas del Desarrollo. Revista Latinoamericana de Economía, vol. 37, núm. 146, julio-septiembre, 2006, pp. 79-86

Universidad Nacional Autónoma de México

Distrito Federal, México

Disponible en: http://www.redalyc.org/articulo.oa?id=11820858004

- Cómo citar el artículo

- Número completo

- Más información del artículo

Página de la revista en redalyc.org

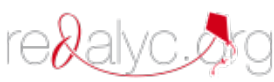

Sistema de Información Científica

Red de Revistas Científicas de América Latina, el Caribe, España y Portugal Proyecto académico sin fines de lucro, desarrollado bajo la iniciativa de acceso abierto 


\section{EXPORTACIONES, TÉRMINOS DE INTERCAMBIO Y CRECIMIENTO ECONÓMICO DE BRASIL Y MÉXICO, DE 1960 A 2002: UN ANÁLISIS COMPARATIVO*}

\section{Carlos Alberto Fraga Castillo** Juan Carlos Moreno-Brid***}

Fecha de recepción: 30 de noviembre de 2005. Fecha de aceptación: 24 de abril de 2006.

\section{Resumen}

México y Brasil son las economías más grandes de la región, caracterizadas por una fuerte presencia de la industria. Ambas han tenido, en diferente grado, un cambio importante en su estrategia de desarrollo, pasando de un modelo centrado en la sustitución de importaciones y la intervención del Estado en la economía a otro modelo abierto a la competencia externa y concentrado en las exportaciones como motor de impulso. El propósito de este trabajo es precisamente comparar en qué medida dicha reorientación "hacia el sector externo" se revela en la capacidad de arrastre del sector exportador al resto de la economía. Para ello se evaluan las elasticidades de comercio y-con base en el modelo desarrollado por A.P. Thirlwall- se estima su impacto en la tasa de crecimiento económico de largo plazo.

Palabras clave: crecimiento, restricción, intercambio, exportaciones, balanza.

\section{Abstract}

Mexico and Brazil are the largest economies in the region, characterized by a strong presence of industry. Both of them, in varying degrees, have experienced important changes in their development strategies, moving away from a model centered on import substitution and State intervention in the economy to another model open to external competition and focusing on exports as the driving force. This work proposes comparing precisely to what extent this re-orientation "towards the external sector" is reflected in a capacity to pull up the rest of the economy. For the purpose it evaluates trade elasticities, and-based on A.P. Thirlwall's development model - estimates their impact on long-term economic growth.

Key words: growth, restriction, exchange, exports, balance.

\footnotetext{
* Los nombres de los autores aparecen en orden alfabético. Las opiniones expresadas en este artículo son de exclusiva responsabilidad de los autores y pueden no coincidir con las instituciones donde laboran.

** Profesor investigador de la Unidad Profesional Interdisciplinaria de Ingeniería y Ciencias Sociales y Administrativas (UPIICSA) del Instituto Politécnico Nacional (IPN). Correo electrónico: carlos fraga@hotmail.com

*** Coordinador de Investigaciones de la Comisión Económica para América Latina y el Caribe (CEPAL oNU), subsede México. Correo electrónico: juancarlos.moreno@cepal.org
} 


\section{CARLOS ALBERTO FRAGA CASTILLO Y JUAN CARLOS MORENO-BRID}

\section{Résumé}

Le Mexique et le Brésil sont les économies les plus grandes de la région, caractérisées par une forte présence de l'industrie. Toutes deux ont eu, à un degré différent, un changement important dans leur stratégie de développement, en passant d'un modèle centré sur la substitution d'importations et dans l'intervention de l'Etat dans l'économie à un autre modèle ouvert à la concurrence externe et centré sur les exportations comme moteur d'impulsion. Le but de ce travail est justement de comparer dans quelle mesure cette réorientation, "vers le secteur externe", se reflète sur la capacité d'entraînement du secteur exportateur sur le reste de l'économie. Dans ce but, on évalue les élasticités de commerce - par rapport au modèle développé par A.P. Thirlwall-, on fait une estimation de son impact sur le taux de croissance économique au long terme.

Mots-cléfs: croissance, restriction, échange, exportation, balance.

\section{Resumo}

Tanto a economia mexicana quanto a brasileira são as maiores da região, caraterizadas por uma forte presença na indústria Ambas, em diferente grau, sofreram uma mudança importante nas suas estratégias de desenvolvimento, passando por um modelo centrado na substituição de importações e a intervenção do Estado na economia a um outro modelo aberto à competência externa e concentrado nas exportações como motor de impulso. O propósito deste trabalho é justamente compararmos em que medida tal reorientação "direcionada ao setor externo", observa-se na capacidade de levamento do setor de exportações ao resto da economia. Para tal objetivo avaliam-se as elasticidades do comércio e —baseado no modelo desenvolvido por A.P. thirlwall-calcula-se o seu impacto na taxa de crescimento económico de longo prazo. Palavras chave: crescimento, restrição, intercâmbio, exportações, balança. 
Introducción: hechos estilizados del desempeño económico de México y de Brasil durante 1960-2002

$\mathrm{E}$

n el comportamiento de ciertas variables fundamentales, tanto de la economía mexicana como de la brasileña, encontramos diferencias significativas. Al examinar el periodo de 1960 a 2002, observamos que en promedio el producto interno bruto (PIB) real de Brasil fue casi dos veces superior al de México. En el mismo lapso, México mantuvo sus exportaciones en 1.6 veces por arriba de las de Brasil, sin embargo, ha requerido sostener el nivel de sus importaciones casi al doble del que ha demandado este país. De tal forma que al relacionar las importaciones con las exportaciones, Brasil se ubicó en mejor posición, ya que la relación fue de uno a uno, mientras que la de México fue tal que las importaciones sumaron casi dos veces el valor de sus exportaciones reales.

A pesar de la existencia de estas diferencias, la experiencia del crecimiento económico de ambos países ha sido bastante similar (Maddison, 1992:4). En las décadas de los sesenta y setenta, tanto Brasil como México crecieron a tasas elevadas, las cuales oscilaron entre $6 \%$ y $7 \%$ de promedio anual. De 1982 a 1990, las dos naciones disminuyeron significativamente su crecimiento, Brasil obtuvo $1.6 \%$ y México no alcanzó ni el 1\%. Por arriba de éstos se elevaron los porcentajes de crecimiento de 1990 a 2000 en los dos países, sólo que México se sostuvo casi a dos puntos porcentuales por arriba de Brasil (véase Cuadro 1 del Apéndice estadístico).

De 1960 a 1981, ambos mantuvieron buen desempeño en su crecimiento, salvo la caída del PIB observada en Brasil en 1972. Este auge prolongado se vio severamente interrumpido por una crisis de deuda externa y de balanza de pagos. A partir de entonces, como se observa en la Gráfica 1, los dos países experimentaron intervalos cortos de expansión con graves caídas en el producto real. En promedio, de 1982 a 2002 el crecimiento fue menor a 2\% (véase Cuadro 1 del Apéndice estadístico), tales tasas de crecimiento fueron comparativamente inferiores a las que se registraron en los años anteriores.

Un componente importante del PIB se forma con las exportaciones. En el Cuadro 1 del Apéndice estadístico observamos que de 1960 a 1981 fue similar el curso que siguió el crecimiento promedio de las exportaciones reales en ambos países. En los setenta aumentó dich crecimiento en comparación con los sesenta, debido a las ventajas que ofreció en la segunda mitad de esa década el mercado petrolero internacional. Tan sólo el crecimiento en el subperiodo 1976 a 1980 fue para Brasil de $11.1 \%$ y para México de 17.4\%, cifras que superan sus correspondientes promedios de los setenta. Situación diferente ocurrió en la mayor parte de los años ochenta, ya que de 1982 a 1990 se contrajo la actividad

\section{Desaarrollo}




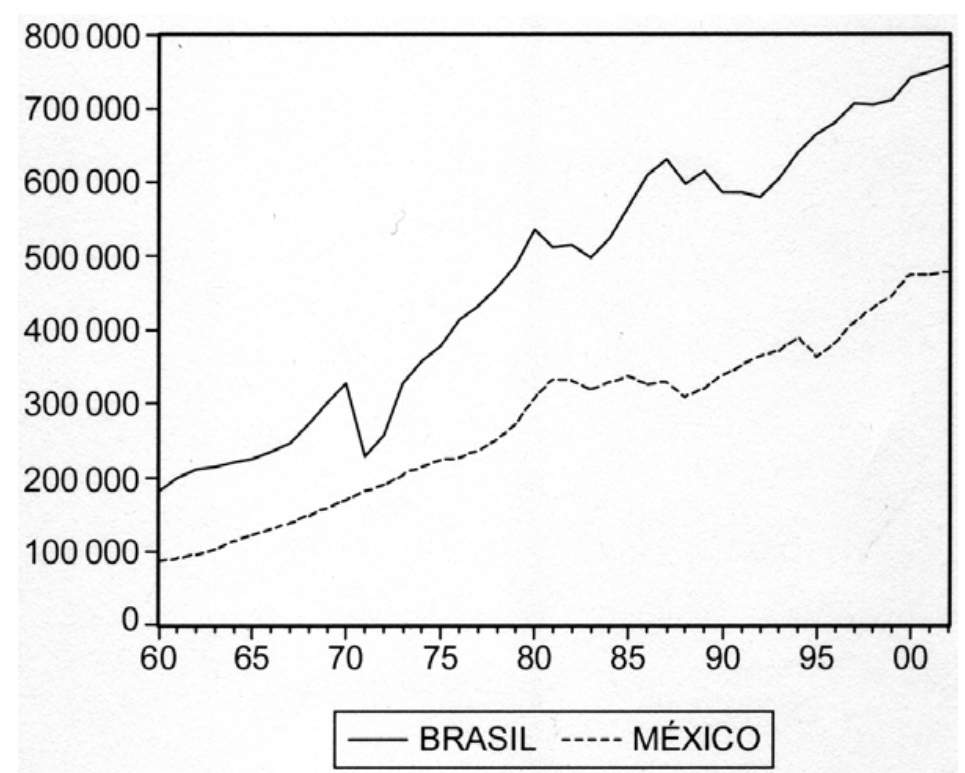

Gráfica 1. PIB de México y Brasil de 1960 a 2002 (millones de dólares a precios constantes de 1995).

Fuente: CEPAL, Cuadernos Estadísticos sobre Crecimiento Económico y Anuarios Estadísticos América Latina y el Caribe.

exportadora de los dos países. Los efectos más nocivos se observaron en el caso de México, que pasó de un crecimiento promedio de 13.1 en los setenta, a 5.7 en ese periodo. Brasil, por su parte, sufrió un retroceso de sólo dos puntos porcentuales, pasando de 9.3 en los setenta, a 7.3 también en ese periodo.

Las cifras del Cuadro 1 del Apéndice estadístico denotan que en los años noventa cambió el rumbo paralelo que habían seguido, en los años anteriores, las exportaciones reales de los dos países. En relación al subperiodo 1990-2002, Brasil observó una reducción en sus exportaciones, mientras que México las incrementó notablemente. La causa de ese fenómeno se encuentra en el papel que jugaron las exportaciones manufactureras, según el informe preliminar de la CEPAL correspondiente a 1999. Se señala que a diferencia de otros productos exportados por la región, "distinta es [...] la suerte de los productos manufacturados exportados por México [...] que se dirigen a los mercados mundiales, sobre todo a Estados Unidos. Las ventas de estos productos se vieron beneficiadas por la expansión de la capacidad productiva de las industrias maquiladoras y el buen ritmo de la economía estadounidense que se estima en 4\% en el año" (CEPAL, 1999:14).

La Gráfica 2 muestra que el crecimiento de las exportaciones reales de los dos países fue relativamente estable durante los primeros diecisiete años del periodo. Aunque con un comportamiento variado desde finales de los setenta hasta principios de los noventa, en ambas naciones la rapidez con que crecieron las exportaciones fue mayor en comparación 


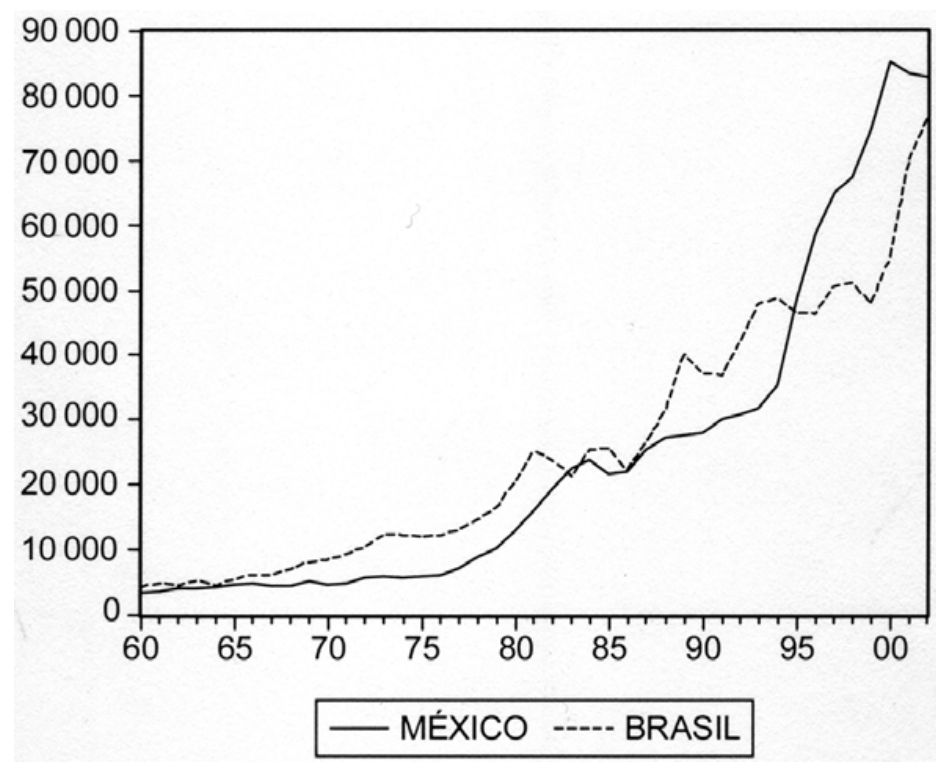

Gráfica 2. Exportaciones reales de México y Brasil de 1960 a 2002 (millones de dólares a precios constantes de 1995).

Fuente: CEPAL, Cuadernos Estadísticos de la Balanza de Pagos, Anuarios Estadísticos de América Latina y el Caribe y Banco de México, Indicadores del Sector Externo.

a los años anteriores. De 1990 a 2000, México creció a mayor velocidad en sus exportaciones que Brasil.

Las gráficas 1 y 2 revelan que en ciertos intervalos ha existido una relación entre el crecimiento de las exportaciones y el del PIB en ambos países. Tal fenómeno se observa, para el caso de Brasil, en el subperiodo 1971-1979, así como el de 1992 hasta 2000. Para México, ocurre de 1976 a 1982, así como de 1994 a 2002.

En la Gráfica 3, es notable que en los últimos años aumentó la presencia de las exportaciones en la producción interna real de las dos naciones. Desde 1982 la participación de las exportaciones mexicanas en el PIB fue mayor que en el caso de Brasil. El acelerado ascenso que registraron las exportaciones mexicanas desde 1994 se debió al buen comportamiento del sector manufacturero.

Se sabe que si bien las importaciones no impulsan la tasa de crecimiento del PIB, en un futuro cercano su comportamiento puede afectar la expansión o contracción de la producción interna de un país. Examinando las importaciones reales tanto de Brasil como de México, representadas en la Gráfica 4, observamos que de 1960 a 1997 siguieron un camino muy semejante; en la mayor parte de esos años el valor real de las compras al exterior de México estuvo ligeramente por arriba del de Brasil. Esa distancia se amplía (véase Gráfica 5) cuando las importaciones reales como proporción del PIB mexicano

\section{Desararrollo}




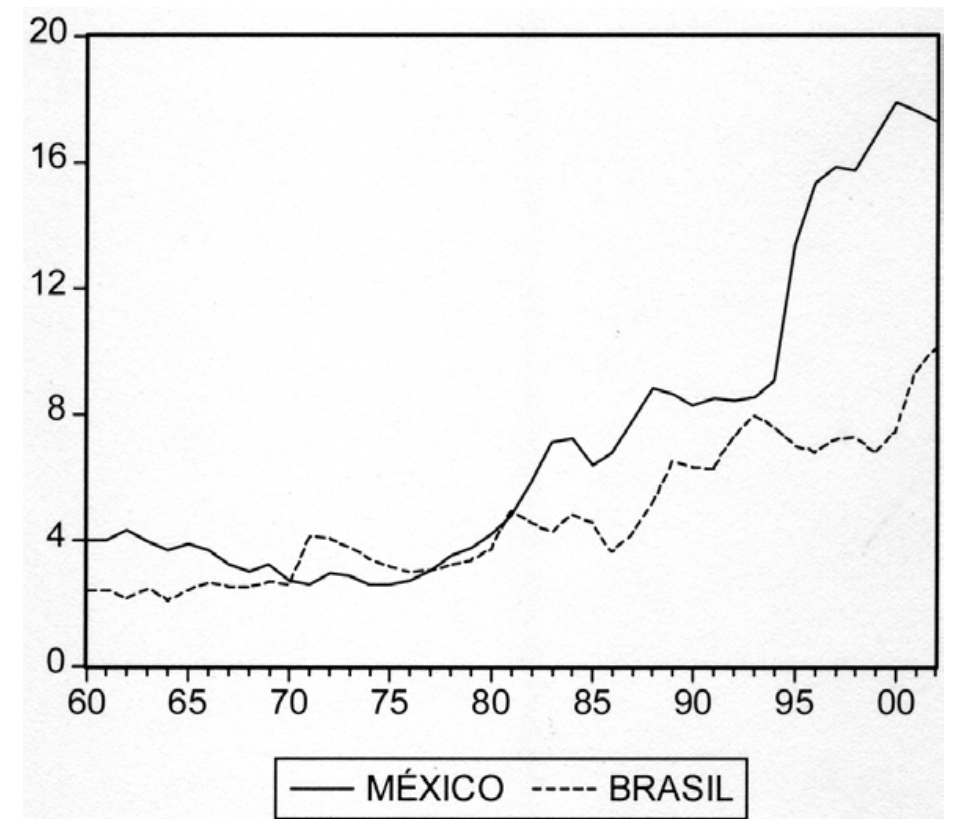

Gráfica 3. Exportaciones reales de Brasil y México como proporción del PIB (1960-2002).

Fuente: $\quad$ CEPAL, Cuadernos Estadísticos de la Balanza de Pagos y Anuarios Estadísticos de América Latina y el Caribe.

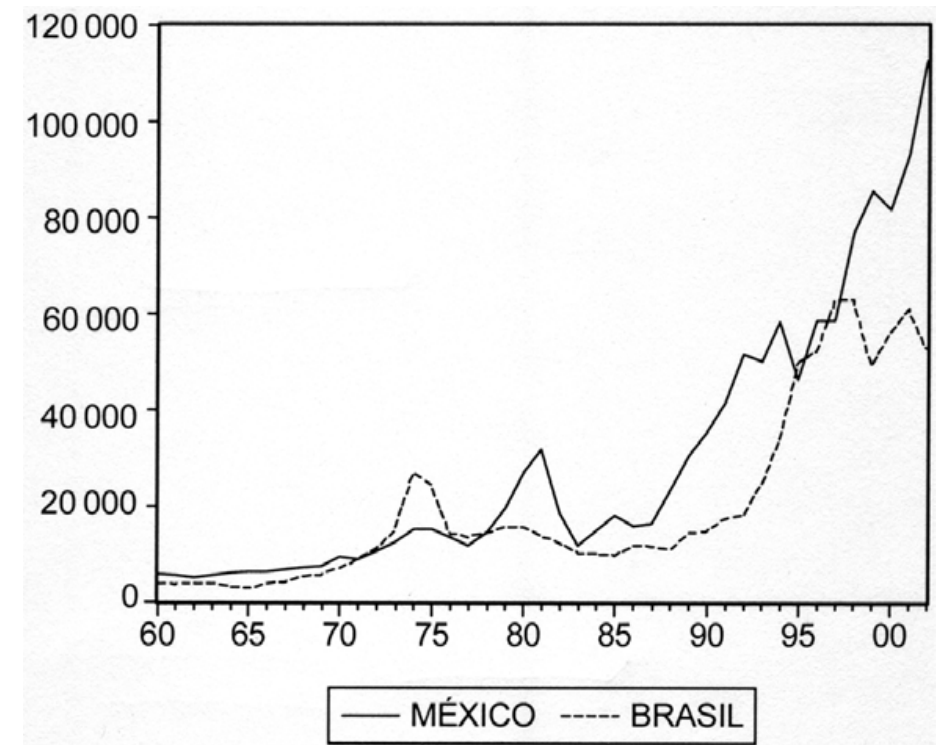

Gráfica 4. Importaciones reales de México y Brasil de 1960 a 2002 (millones de dólares de 1995).

Fuente: CEPAL, Cuadernos Estadísticos de la Balanza de Pagos y Anuarios Estadísticos de América Latina y el Caribe. 


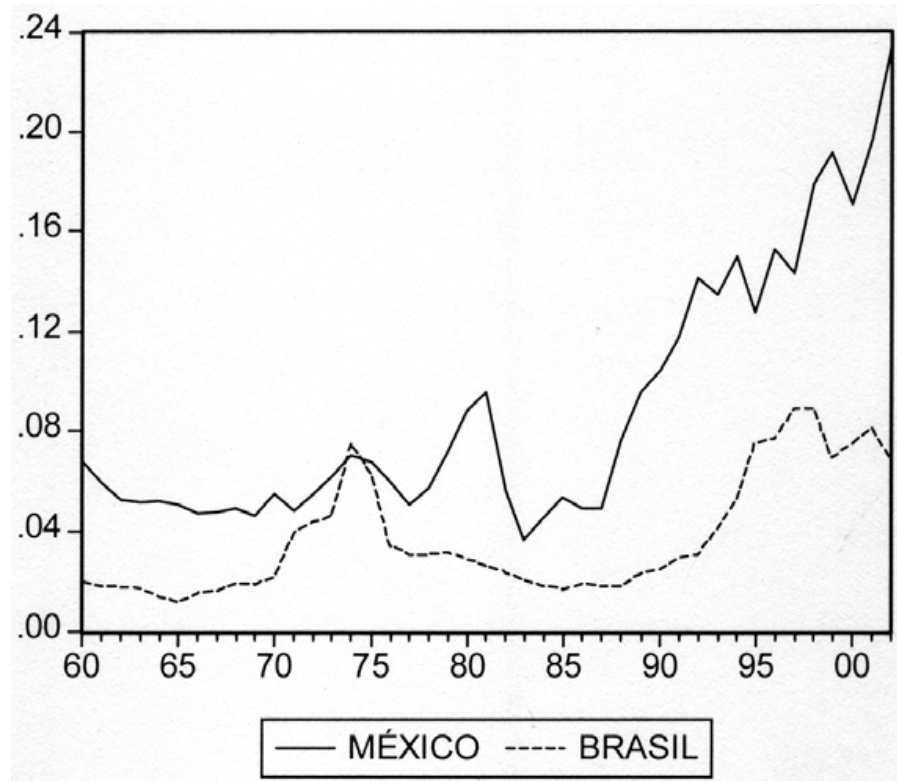

Gráfica 5. Importaciones reales de México y Brasil (como proporción del PIB).

Fuente: CEPAL, Cuadernos Estadísticos de la Balanza de Pagos y Anuarios Estadísticos de América Latina y el Caribe.

supera en mucho durante todo el periodo a las de Brasil, lo cual implica que México destina más de su ingreso al pago de importaciones que éste.

Otra variable de interés para explicar el crecimiento del PIB la conforman los términos de intercambio. El Cuadro 1 del Apéndice estadístico muestra que en algunos subperiodos se presenta una asociación entre las tasas de crecimiento promedio de los términos de intercambio y del PIB real. Por ejemplo, de 1960 a 1981 México tuvo un crecimiento favorable y significativo en sus términos de intercambio con una alta tasa de crecimiento en su PIB real. En el mismo periodo, Brasil experimentó un deterioro no muy severo (0.7\%) con una tasa de crecimiento del PIB menor a la de México. Otro fenómeno que se observa en el Cuadro 1 es que durante el periodo 1960-2002 los términos de intercambio fueron muy poco favorables tanto para México (0.7\%) como para Brasil (0.6\%). Tales porcentajes no son muy altos y revelan cierto grado de estabilidad en el largo plazo y también en lapsos cortos como los primeros y últimos años del periodo (véase Gráfica 6).

Aunque Prebisch (1951) observó una tendencia al deterioro de los términos de intercambio, en los trabajos de Thirlwall y McCombie se sostiene que en condiciones de largo plazo dichos términos permanecen constantes (Mc Combie y Thirlwall, 2000:261). Ello ocurre, según estos autores, porque las pérdidas o las ganancias que puede tener un país en sus términos de intercambio en periodos cortos se compensarán unas con otras en el largo plazo, de tal forma que los precios relativos se aproximen a la unidad.

\section{Desaarrollo}




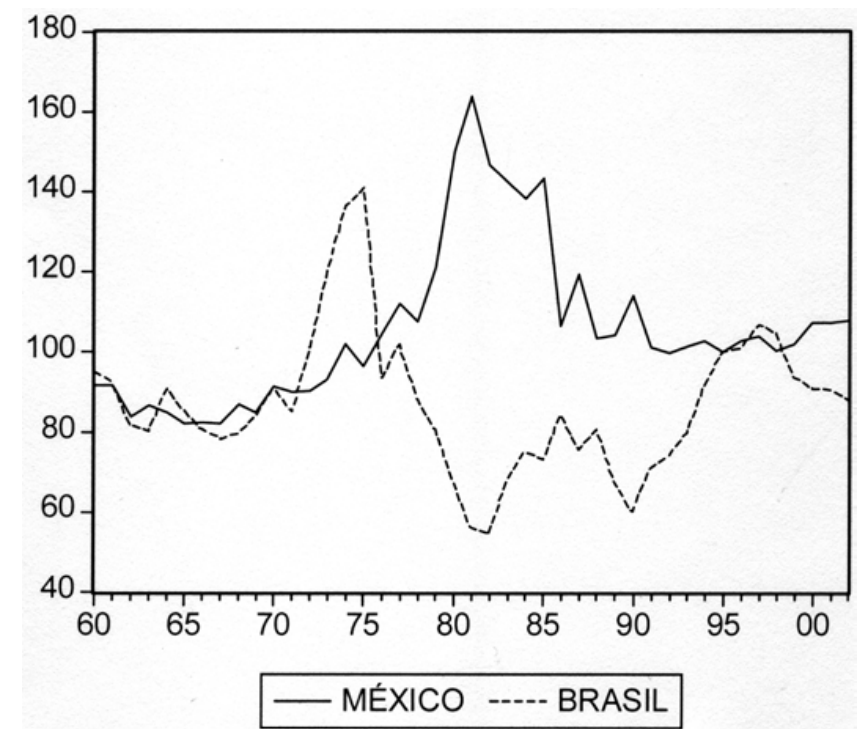

Gráfica 6. Términos de intercambio de Brasil y México: 1960-2002 (1995=100).

Fuente: CEPAL, Cuadernos Estadísticos de la Balanza de Pagos y Anuarios Estadísticos de América Latina y el Caribe.

Entre las teorías que explican el crecimiento económico determinado por el comercio exterior se encuentra la de Thirlwall. Una vez desarrollada, emplearemos el análisis de integración y cointegración con el objeto de estimar una relación de largo plazo entre el crecimiento del PIB real, las exportaciones y los términos de intercambio y, por último, presentaremos las conclusiones finales.

\section{Marco teórico}

El modelo en cuestión tiene sus fundamentos en los trabajos de Harrod (1939) y fue desarrollado por Thirlwall (1979). Dentro de la literatura económica se le conoce como modelo de crecimiento económico con restricción de balanza de pagos (modelo CRBP). Para su desarrollo, hemos tomado como base el trabajo "Términos de intercambio, exportaciones y crecimiento económico en América Central: una visión de largo plazo", concebido por Pérez y Moreno-Brid, debido a que dicho artículo posee la virtud de considerar los términos de intercambio actuando dentro del modelo y no supone que los términos de intercambio permanezcan constantes, como ocurre en la aplicación del modelo de Thirlwall hecha para el caso de México por Moreno-Brid (1998) y (1999), y para Brasil realizada por Holland (1998) y Barbosa (2002).

En la estructura analítica del artículo los autores presentan el modelo CRBP en su versión más generalizada formulada tanto por Thirlwall como Hussain (1982) en las siguientes seis ecuaciones: 


$$
p x+e f^{*}=p^{*} e m
$$

La ecuación (1) expresa la identidad de la cuenta de la balanza de pagos, donde $p$ representa el precio de los bienes internos o de exportación en moneda local, $x$ las exportaciones reales, $p$ el precio de las importaciones en moneda extranjera, $m$ las importaciones reales, $f$ el déficit en cuenta corriente de la balanza de pagos en unidades de moneda externa, y $e$ es el tipo de cambio nominal expresado en unidades de moneda local por unidades de la moneda extranjera.

$$
\begin{array}{r}
\theta=p \times /\left(p x+e f^{*}\right) \\
n=p-p^{*}-e
\end{array}
$$

Las ecuaciones (2) y (3) son identidades de definición introducidas para simplificar la notación algebraica, en la cual $\theta$ expresa la participación inicial de las exportaciones en el flujo total de divisas medidas en precios corrientes, y $n$ se define como la tasa de cambio de los términos de intercambio.

$$
\begin{gathered}
\theta(\hat{\mathrm{p}}+\hat{\mathrm{x}})+(1-\theta)(\hat{\mathrm{f}} * \hat{\mathrm{e}})=\hat{\mathrm{p}}^{*}+\hat{\mathrm{e}}+\hat{\mathrm{m}} \\
\hat{\mathrm{x}}=\eta \hat{\mathrm{n}}+\pi \hat{\mathrm{w}}, \eta<0, \pi>0 \\
\hat{\mathrm{m}}=\phi \hat{\mathrm{n}}+\zeta \hat{\mathrm{y}}, \phi<0, \xi>0
\end{gathered}
$$

Por propósitos de notación, el símbolo $z$ denota tasas de crecimiento.

La ecuación 4 se obtiene diferenciando la ecuación 1 con respecto del tiempo y utilizando la notación de la expresión (2). Las ecuaciones (5) y (6) especifican las tasas de cambio de la demanda de exportaciones reales $\hat{x}$ y de importaciones reales $\hat{m}$, ambas corresponden a funciones convencionales de demanda de exportaciones e importaciones con elasticidades constantes en relación con los ingresos y los precios, donde $\eta$ y $\pi$ son las elasticidades precio e ingreso de las exportaciones, $\phi$ y $\xi$ son las elasticidades precio e ingreso de las importaciones, respectivamente, $\hat{y}$ representa la tasa de crecimiento de los niveles del ingreso nacional en términos reales y $\hat{w}$ la del ingreso del exterior. La solución de las ecuaciones (1) a la (6) está dada por la siguiente ecuación:

$$
y_{b}=\frac{\theta \pi \hat{\mathrm{w}}+(1-\theta)\left(\hat{\mathrm{f}}^{*}+\hat{\mathrm{e}}-\hat{\mathrm{p}}\right)+(\theta \eta+\phi+1)(\mathrm{n})}{\xi}
$$


La ecuación (7) expresa que la tasa de crecimiento de largo plazo del ingreso nacional $\hat{y_{b}}$ se determina por las tasas de crecimiento del ingreso externo $\hat{w}$, de flujos externos de capital en términos reales $\hat{\mathrm{f}}+\hat{\mathrm{e}}-\hat{\mathrm{p}} \mathrm{y}$ de los términos de intercambio $\hat{n}$, con los coeficientes de ponderación de los tres elementos dados por las elasticidades con respecto del precio e ingreso de las exportaciones e importaciones y por la participación de las exportaciones en el flujo total de divisas.

Si suponemos que $\theta=1$, en la ecuación (7) obtenemos la tasa de crecimiento económico consistente con el supuesto de que en el largo plazo la cuenta corriente de la balanza de pagos debe ser cero

$$
\mathrm{y}_{\mathrm{b}}=\frac{\hat{\hat{\mathrm{w}}+(\eta+\phi+1)(\mathrm{n})}}{\xi}
$$

Si sustituimos en la ecuación (8) la expresión $\pi w$ de la ecuación (5), obtenemos la tasa de crecimiento económico con restricción de balanza de pagos $y_{b}$ como una combinación lineal de la tasa de crecimiento de las exportaciones $x$ y de los términos de intercambio $n$ :

$$
\mathrm{y}_{\mathrm{b}}=\frac{\hat{\mathrm{x}}+(\mathrm{f}+1) \hat{\mathrm{n}}}{\xi}
$$

Si los términos no muestran variaciones significativas en el largo plazo, la tasa de crecimiento económico con restricción de balanza de pagos está dada por la tasa de crecimiento de las exportaciones y la elasticidad ingreso de las importaciones, en la expresión conocida como ley de Thirlwall (Pérez, y Moreno-Brid, 1999:436-437).

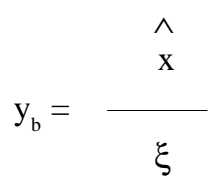

El modelo plantea un problema central: la existencia de factores que pueden restringir el crecimiento de la demanda agregada y, por consecuencia, del crecimiento económico. El comercio exterior es el factor al que Thirlwall da mayor relevancia como limitante del crecimiento, y según Fujii (2000), en una economía abierta la restricción de demanda más importante proviene de la necesidad de mantener el equilibrio en la cuenta corriente de la balanza de pagos (Fujii, 2000:17).

\section{Desarrollo}


Sin embargo, en el modelo el comercio exterior no es el único factor de demanda agregada que puede restringir el crecimiento hasta un cierto punto, también es uno de los elementos propulsores del crecimiento de una economía.

En ese mismo sentido lo han planteado Pérez y Moreno-Brid cuando explican que la perspectiva analítica introducida por Thirlwall y sus colegas, en contraposición al énfasis que hace la teoría neoclásica acerca de la oferta de factores de producción, identifica la demanda agregada y la capacidad del comercio exterior como las fuerzas que impulsan y atan el crecimiento económico (Pérez y Moreno-Brid, 1999:435).

Davidson (1997) ha reconocido los aportes teóricos de la ley de Thirlwall como un avance considerable dentro de la escuela poskeynesiana, al asegurar que, en el contexto de la economía global de fines del siglo xx, los economistas poskeynesianos han generalizado el análisis keynesiano de una economía cerrada de los años treinta, ocupándose del crecimiento económico de una nación cuando el comercio exterior es un componente importante de demanda agregada. Una de las más significativas contribuciones hacia esta teoría poskeynesiana de crecimiento endógeno en una economía abierta ha sido la ley de Thirlwall (Davidson, 1997:312).

No sólo han despertado interés las deducciones teóricas a las que ha llegado Thirlwall, sino que también han llamado la atención las conclusiones obtenidas a partir de pruebas modernas de econometría. Así lo ha expresado McCombie cuando afirma que de particular relevancia para las pruebas de la ley de Thirlwall son las considerables implicaciones de las recientes innovaciones en el análisis de las series de tiempo con respecto de la integración y cointegración (McCombie, 1997:335).

La ecuación (10) del modelo CRBP ha servido para todas las pruebas econométricas que procuran verificar la ley de Thirlwall. Sin embargo, nos concentraremos en la ecuación (9) que contiene los efectos de los términos de intercambio y las exportaciones sobre la tasa de crecimiento. Cabe aclarar que existe un interesante debate sobre lo que Perrotini llama el "supuesto de neutralidad de los precios relativos" (Perrotini, 2002:vi), es decir, de la inclusión o exclusión que hacen los autores de los términos de intercambio en el modelo de Thirlwall. Nos parece válido inclinarnos por los autores que vinculan los términos de intercambio y el crecimiento económico en dicho modelo, puesto que la discusión teórica y empírica de un problema es la fuente que enriquece a una ciencia y no así las verdades absolutas. Además, el problema planteado por Prebisch (1951) permanece, ya que según la UNCTAD (2002) en años recientes los países que producen y exportan bienes manufacturados, cuya fabricación registra baja proporción en el empleo de tecnología y alta proporción en el uso de mano de obra, han tenido una tendencia decreciente en sus términos de intercambio (UNCTAD, 2002:117-120).

\section{Desaarrollo}




\section{Verificación empírica para México y Brasil: 1960-2002}

Para el ejercicio empírico, la ecuación (9) se formula en su correspondiente expresión funcional en primeras diferencias:

$$
\Delta \log \left(\mathrm{y}_{\mathrm{b}}\right)=\alpha \Delta \log (\mathrm{x})+\beta \Delta \log (\mathrm{n})+\gamma
$$

en la cual, por cuestiones de notación, se introducen dos identidades: $\alpha=1 / \xi, \beta=(\phi+1) / \xi$ y $\gamma$ es un término constante.

Para el análisis de cointegración se requiere, en primera instancia, investigar si cada una de las series de tiempo es o no estacionaria. Las pruebas Dickey-Fuller (DF) y DickeyFuller Aumentada (ADF, por sus siglas en inglés) ${ }^{1}$ son fundamentales para tal efecto. La aceptación de la hipótesis nula conocida como $\mathrm{H}_{0}: \rho=1$, la cual determina la existencia de una raíz unitaria en cada una de las series de tiempo y, por consecuencia, indica el carácter no estacionario de dichas series. Si se rechaza la hipótesis nula y se acepta la alternativa conocida como $\mathrm{H}_{1}: \rho<1$, no existe raíz unitaria y las series son estacionarias. Adicionalmente, emplearemos esa prueba con el fin de establecer el orden de integración de las variables, es decir, el número de veces que debe obtenerse la primera diferencia de la variable para lograr series estacionarias.

En el Cuadro 1 (siguiente página), el asterisco señalado en tres valores indica que las series pasan la prueba de raíz unitaria, aunque no se les aplique la primera diferencia. A pesar de eso, no se puede determinar el orden de integración porque al menos dos valores de esas series no la pasan. El resto de las series tampoco, por lo que se procedió a calcular la prueba ADF aplicando primeras diferencias a las series. En el mismo cuadro, todos los valores de las series en primeras diferencias sean con constante, con constante y tendencia o sin constante ni tendencia pasan la prueba. Por lo tanto, todas las series están integradas en orden $\mathrm{I}(1)$.

Una vez que se ha determinado el orden de integración y que constatamos que todas las variables lo tienen, la práctica establecida indica que si las series son estacionarias y del mismo orden, se procede a calcular la prueba de cointegración de Johansen (Cuthbertson, 1992:153-154).

Para conocer el número de rezagos óptimo, calculamos un vector autorregresivo e investigamos su nivel óptimo de rezagos por medio de los criterios que se muestran en el Cuadro 2.

1 La especificación para llevar a cabo la prueba Dickey Fuller Aumentada para cualquier variable, por ejemplo $X_{t}$, consiste en definir la siguiente ecuación:

$$
\Delta \log X_{t}=\alpha \log X_{t-1}+\sum_{j-1}^{p-1} \beta \Delta \log X_{t-j}+\varepsilon_{t}
$$


Cuadro 1

Prueba Dickey-Fuller Aumentada (ADF) aplicada al producto interno bruto real, las exportaciones y los términos de intercambio de México y Brasil (1960-2002)

\begin{tabular}{|c|c|c|c|c|c|c|}
\hline & $\begin{array}{rl}L & o g \\
\text { Nivel } & \end{array}$ & $\begin{array}{l}P \quad I \quad B \\
\quad \\
\quad l a . \Delta\end{array}$ & $\begin{array}{l}L O g \\
\text { Nivel }\end{array}$ & $\begin{array}{l}X \\
\text { la. } \Delta\end{array}$ & $\begin{array}{l}L o g \\
\text { Nivel }\end{array}$ & $\begin{array}{l}T I \\
1 a . \Delta\end{array}$ \\
\hline \multicolumn{7}{|l|}{ México } \\
\hline \multicolumn{7}{|l|}{ ADF calculada: } \\
\hline Con constante & $-3.2 *(0)$ & $-3.0(0)$ & $-0.03(0)$ & $-3.9(0)$ & $-1.9(0)$ & $-7.5(0)$ \\
\hline Con constante y tendencia & $-1.8(1)$ & $-5.0(0)$ & $-1.8(3)$ & $-4.0(0)$ & $-1.69(0)$ & $-7.4(0)$ \\
\hline Sin constante ni tendencia & $2.8(0)$ & $-2.8(0)$ & $-2.5^{*}(0)$ & $-2.9(0)$ & $-0.8(0)$ & $-7.5(0)$ \\
\hline \multicolumn{7}{|l|}{ Brasil } \\
\hline Con constante & $-1.5(0)$ & $-6.3(1)$ & $2.2(7)$ & $-6.0(1)$ & $-2.0(0)$ & $-5.6(0)$ \\
\hline Con constante y tendencia & $-2.1(1)$ & $-5.9(1)$ & $-3.1(1)$ & $-5.1(6)$ & $-2.0(0)$ & $-5.5(0)$ \\
\hline Sin constante ni tendencia & $2.6(0)$ & $-5.5(0)$ & $4.1^{*}(2)$ & $-0.6(7)$ & $-0.2(0)$ & $-5.6(0)$ \\
\hline
\end{tabular}

Los valores ADF calculada se procesaron en E-views versión 4.1. El programa presentó como valores críticos a $5 \%$ los siguientes: con constante, en nivel y en primera diferencia -2.9 ; con constante, tendencia, en nivel y en primera diferencia 3.5; sin constante, ni tendencia, en nivel y en primera diferencia -1.95. El valor que aparece entre paréntesis indica el nivel de rezagos óptimo, siguiendo el criterio de Akaike.

Cuadro 2

Criterios de selección de rezagos

\begin{tabular}{lcccc}
\hline & $\begin{array}{c}\text { De información } \\
\text { de Akaike }\end{array}$ & $\begin{array}{c}\text { Error } \\
\text { de pronóstico }\end{array}$ & $\begin{array}{c}\text { De información } \\
\text { de Schwarz }\end{array}$ & $\begin{array}{c}\text { De información } \\
\text { de Hannan-Quinn }\end{array}$ \\
\hline México & 2 & 2 & 2 & 1 \\
Brasil & 1 & 1 & 1 & 1 \\
\hline
\end{tabular}

Los cuatro criterios coinciden en la introducción de un rezago al vector autorregresivo (VAR) formado por las tres series.

Cuadro 3

Prueba de cointegración de Johansen

\begin{tabular}{clrrr}
\hline País & & $\lambda_{\max }$ & \multicolumn{1}{c}{$5 \%$} & \multicolumn{1}{c}{ VEC } \\
\hline \multirow{2}{*}{ México } & Ho & & & \\
& $\mathrm{r}=0$ & 30.56 & 24.31 & $\Delta \mathrm{LGDPM}=0.42 \Delta \mathrm{LXM}+1.91 \Delta$ LTIM \\
& $\mathrm{r}=1$ & 10.42 & 12.53 & \\
& $\mathrm{r}=2$ & 1.96 & 3.89 & \\
& & & & \\
Brasil & Ho & & & \\
& $\mathrm{r}=0$ & 23.10 & 20.97 & $\Delta \mathrm{LGDPB}=0.51 \Delta \mathrm{LXB}-0.44 \Delta \mathrm{LTIB}$ \\
& $\mathrm{r}=1$ & 6.08 & 14.07 & \\
& $\mathrm{r}=2$ & 1.04 & 3.76 & \\
\hline
\end{tabular}

\section{Desararrollo}


Según la prueba de cointegración de Johansen, presentada en el Cuadro 3, existe una relación lineal en primeras diferencias entre las tasas de crecimiento del PIB, las exportaciones y los términos de intercambio para cada país. La raíz característica máxima $\left(\lambda_{\max }\right)$ a 5\% señala que existe al menos un vector de cointegración (VEC) para cada caso. Los coeficientes $\alpha$ y $\beta$ correspondientes a la ecuación de Brasil indican que las exportaciones reales, más que los términos de intercambio, determinaron la tasa de crecimiento del producto real de largo plazo.

Aunque los coeficientes de la ecuación que representa el crecimiento económico de México muestran que los términos de intercambio, más que las exportaciones, explican la tasa de crecimiento de largo plazo, al calcular las elasticidades de cada variable con respecto de la tasa de crecimiento del producto real por el método de mínimos cuadrados ordinarios, encontramos que la de mayor peso corresponde a la de las exportaciones con $3.61 \%$ y la de los términos de intercambio con 3.22\%. De esta manera, también en el caso de México, la tasa de crecimiento del piB real de largo plazo estuvo determinado en mayor medida por las exportaciones que por los términos de intercambio.

De los vectores de cointegración del Cuadro 3 podemos calcular las elasticidades ingreso de las importaciones. De la identidad algebraica $\alpha=1 / \xi$, expresada en la ecuación 11 y que se deriva del modelo de Thirlwall, despejamos la elasticidad ingreso de las importaciones $(\xi)$, y obtenemos $\xi=1 / \alpha$.

Los resultados del Cuadro 4 denotan valores altos en la elasticidad ingreso de las importaciones de ambos países e indican que ante aumentos en el ingreso nacional, la sensibilidad de la demanda mexicana por productos externos es ligeramente mayor que la de Brasil, lo cual implica ceteris paribus —y según la versión más sencilla del modelo de Thirlwall— que México, en comparación con Brasil, quizá tenga mayor restricción a su crecimiento proveniente de la balanza de pagos. ${ }^{2}$

Ello, claro y aun en este simple modelo, dependerá del dinamismo de las exportaciones. Como muestra el cuadro, incluso suponiendo válido el estimado de una elasticidad ingreso de las importaciones mayor en el caso mexicano, su mejor desempeño de las exportaciones permite compensarla de tal forma que la tasa de expansión económica de largo plazo para el periodo de análisis es superior.

\section{Conclusiones}

En el presente artículo se analizó la relación entre el crecimiento del producto interno bruto real con el de las importaciones y exportaciones reales y el de los términos de

2 La diferencia entre las elasticidades -ingreso de largo plazo de las importaciones de ambos países aquí estimadas es relativamente pequeña. En consecuencia, habría que aplicar en el futuro una prueba estadística sobre los parámetros estimados a fin de poder concluir que, en efecto, la elasticidad ingreso de México, para el periodo de análisis, es significativamente superior a la de Brasil. 
Cuadro 4

Elasticidad-ingreso de las importaciones

\begin{tabular}{ll}
\hline País & $\mathrm{x}$ \\
\hline México & 2.3 \\
Brasil & 2 \\
\hline
\end{tabular}

Cuadro 5

\begin{tabular}{lccc}
\hline País & Crecimiento PIB observado & Crecimiento exportaciones observadas & PIB estimado \\
\hline México & 4.3 & 8.3 & 4.7 \\
Brasil & 3.8 & 7.6 & 3.6 \\
\hline
\end{tabular}

Tasa medias anuales de crecimiento observado del PIB real y de las exportaciones reales, y estimado del PIB real según el modelo simple de Thirlwall: 1960-2002

Fuente: cálculos de los autores.

intercambio, ocurrida en México y Brasil de 1960 a 2001. Se aplicaron técnicas de cointegración al modelo CRBP - en la versión que establece una dependencia lineal entre la tasa de crecimiento del producto real con las exportaciones y los términos de intercambio—en ambos países.

Los coeficientes de los vectores de cointegración indicaron que son las exportaciones y no tanto los términos de intercambio los que influyeron en la tasa de crecimiento de largo plazo de Brasil y, en el caso de México, las elasticidades de cada variable con respecto del producto mostraron la misma tendencia que el primero. Los valores que asumieron las elasticidades ingreso de las importaciones, que en términos matemáticos son los inversos del parámetro $\alpha$, resultaron altos tanto para México como para Brasil.

Esos resultados sugieren que la política económica de los dos gobiernos debe buscar la forma para disminuir las elasticidades ingreso de las importaciones o, bien, aumentar el dinamismo de sus exportaciones. Para ello, bien puede ser necesario revisar las políticas comerciales y de promoción de la competitividad e innovación en cada caso. Finalmente, recordamos que en este trabajo, por problemas de falta de información de largo plazo, hemos excluido el caso de las maquiladoras en México. Su análisis es parte de un trabajo en curso de los autores que esperamos poder reportar en el futuro cercano. Igualmente queremos subrayar que, asimismo, por cuestiones de tiempo y espacio, en el presente artículo no incluimos el análisis del efecto del tipo de cambio real. Dicho análisis, por demás importante, será motivo de una investigación posterior. 


\section{Bibliografía}

Barbosa, N., "The Balance-of-Payments Constraint: From Balance Trade to Sustainable Debt", CEPA Working Paper. núm. 2001-06, Nueva York, New School University, Center For Economic Policy Analysis, 2002.

CEPAL, Anuario estadístico para América Latina, 1970, 1980, 1990, 1999, 2003.

CEPAL, BADEPAG, Cuadernos Estadísticos, núm. 5, Balance de pagos, 1950-1977.

CEPAL, Cuadernos Estadísticos, núm. 3, Crecimiento Económico, 1950-1977

_ Balance Preliminar de la Economía de América Latina. 1980, 1990, 1999.

Cuthbertson, K., S.G. Hall y M.P. Taylor, Applied Econometrics Techniques, The University of Michigan Press, 1992.

Davidson, P., Journal of Poskeynesian Economics, primavera, vol. 19, núm. 5, 1997.

Fujii, G., "La Agricultura, la Agroindustria, y la Restricción Externa al Crecimiento Económico de México", en Problemas del Desarrollo, IECCuNAM, vol. 31, núm. 172, 2000.

Harrod, R., "An Essay in Dynamic Theory", Economic Journal, marzo, 1939.

Holland, M., O. Canuto y X. Clesio, "Taxas de Câmbio, Elasticidades-renda e Saldo Comercial na Economia Brasileira", Revista Brasileira da Economia, vol. 52, núm. 2, 1998.

McCombie, J. y A. Thirlwall, Economic Growth and the Balance of Payments Constraint, St. Martins Press, Kent, 2000

McCombie, J., "On the Empirics of Balance-ofPayments-Constrained Growth", Journal of Poskeynesian Economics, primavera, vol. 19. núm. 5, 1997.
Moreno-Brid, J., "México: Crecimiento económico y restricción de balanza de pagos", en Comercio Exterior, vol. 48, núm. 6, México, 1998.

- "Mexico's Economic Growth and the Balance of Payments Constraint: a Cointegration Analysis", en International Review of Applied Economics, vol. 13, núm. 2, 1999.

Moreno-Brid, J. y E. Pérez, "Balanza de Pagos y Crecimiento Económico en América Central,1950-1996", en Comercio Exterior, 2000.

, "Liberalización Comercial y Crecimiento Económico en Centroamérica", Revista de la CEPAL, núm. 81, CEPAL-ONU, Santiago, 2004.

Perrotini, I., "La Hipótesis de A. P. Thirlwall: Teoría, Crítica y Evidencia Empírica”, versión Preliminar, 2002.

Pérez, E. y J. Moreno-Brid, Terms of Trade, Exports and Economic Growth in Central America: A Long-term View. Banca Nazionale del Lavoro Quartely Review, 1999.

Prebisch, R., Problemas Teóricos y Prácticos del Crecimiento, E/CN. 12/21 Santiago de Chile. 1951.

Thirlwall, A.P., The Balance of Payments as Constraint, as an Explanation of International Growth Rate Differences, Banca Nazionale de Laboro Quarterly Review, 1979.

Thirlwall, A.P. y M. Hussain, The Balance of Payments as Constraint, Capital Flows and Growth Rate Differences Between Developing Countries. Oxford Economic Papers, 1982.

UNCTAD, Trade and development Report, 2002, United Nations, New York and Geneva, 2002. 
Apéndice estadístico

Crecimiento promedio del PIB real, exportaciones, importaciones y términos de intercambio de Brasil y México,* de 1960 a 2002

\begin{tabular}{lrrrrrrrr}
\hline & $\begin{array}{r}1960- \\
2002\end{array}$ & $\begin{array}{r}1960- \\
1981\end{array}$ & $\begin{array}{r}1960- \\
1994\end{array}$ & $\begin{array}{r}1982- \\
2002\end{array}$ & $\begin{array}{r}1960- \\
1970\end{array}$ & $\begin{array}{r}1970- \\
1980\end{array}$ & $\begin{array}{r}1980- \\
1990\end{array}$ & $\begin{array}{r}1990- \\
2002\end{array}$ \\
\hline México & & & & & & & & \\
Exportaciones & 8.3 & 8 & 7.5 & 8.6 & 3.2 & 9.3 & 10.1 & 9.2 \\
Importaciones & 9.1 & 9.3 & 8.9 & 9.2 & 5.2 & 13.9 & 9.6 & 11.5 \\
Términos de intercambio & 0.7 & 2.7 & 0.7 & -1.8 & 0.1 & 5.6 & 0.3 & 0.3 \\
PIB, real & 4.3 & 6.6 & 4.6 & 1.9 & 7 & 6.2 & 2.1 & 3.4 \\
& & & & & & & & \\
Brasil & & & & & & & & \\
Exportaciones & 7.6 & 8.3 & 7.9 & 6.0 & 7.4 & 8.9 & 8.7 & 5.5 \\
Importaciones & 9.2 & 10.2 & 8.9 & 9.3 & 8.2 & 13.5 & 0.3 & 13.9 \\
Términos de intercambio & 0.6 & -0.8 & 0.7 & 2.8 & -0.2 & -0.6 & -1.7 & 2.7 \\
PIB, real & 3.8 & 5.6 & 4.2 & 1.9 & 6.1 & 6.3 & 1.8 & 1.7 \\
\hline
\end{tabular}

Fuente: CEPAL, Cuadernos Estadísticos sobre Crecimiento Económico y Balanza de Pagos; y Anuarios Estadísticos de América Latina y el Caribe. Banco de México indicadores del sector externo. Los valores reales del PIB, las exportaciones y las importaciones fueron calculados a precios constantes de 1995, mientras que para los términos de intercambio el año base fue 1995 (1995=100). 
PIB real, exportaciones reales, importaciones reales de México y Brasil millones de dólares a precios constantes de 1995 y términos de intercambio $(\mathbf{1 9 9 5}=\mathbf{1 0 0})$

\begin{tabular}{|c|c|c|c|c|c|c|c|c|}
\hline Años & $\begin{array}{c}\text { PIB } \\
\text { Brasil }\end{array}$ & $\begin{array}{c}P I B \\
\text { México }\end{array}$ & $\begin{array}{l}\text { Exportacio- } \\
\text { nes reales } \\
\text { de México }\end{array}$ & $\begin{array}{c}\text { Exportacio- } \\
\text { nes reales } \\
\text { de Brasil }\end{array}$ & $\begin{array}{c}\text { Importacio- } \\
\text { nes reales } \\
\text { de Brasil }\end{array}$ & $\begin{array}{l}\text { Importacio- } \\
\text { nes reales } \\
\text { de México }\end{array}$ & $\begin{array}{l}\text { Términos } \\
\text { de Inter. } \\
\text { de Brasil }\end{array}$ & $\begin{array}{c}\text { Términos } \\
\text { de Inter. } \\
\text { de México }\end{array}$ \\
\hline 1960 & 181092.9 & 85763.2 & 3447.2 & 4373.43 & 3655.37 & 5832.64 & 95.02 & 91.83 \\
\hline 1961 & 199754.3 & 89990.8 & 3596.5 & 4838.32 & 3652.54 & 5349.96 & 91.92 & 91.64 \\
\hline 1962 & 210229.4 & 94196.5 & 4098.9 & 4519.76 & 3686.47 & 4951.25 & 81.64 & 83.60 \\
\hline 1963 & 213489.5 & 101719 & 4057 & 5230.27 & 3658.2 & 5289.98 & 80.27 & 86.62 \\
\hline 1964 & 219737.9 & 113616.1 & 4198.5 & 4546.39 & 3070.17 & 5934.77 & 90.92 & 84.79 \\
\hline 1965 & 225728.1 & 120983.9 & 4698.2 & 5441.76 & 2660.25 & 6108.05 & 85.46 & 81.96 \\
\hline 1966 & 234218.9 & 129369.9 & 4740.4 & 6223.06 & 3683.64 & 6131.96 & 80.73 & 82.42 \\
\hline 1967 & 245650.8 & 137480.9 & 4428.4 & 6152.82 & 4073.77 & 6566.77 & 78.270 & 82.14 \\
\hline 1968 & 273091.6 & 148665.7 & 4427.4 & 6857.64 & 5244.17 & 7271.43 & 79.73 & 87.08 \\
\hline 1969 & 300205.7 & 158067.6 & 5099.3 & 8074.64 & 5634.3 & 7354.97 & 84.01 & 84.88 \\
\hline 1970 & 326665.6 & 169007.8 & 4577.3 & 8507.8 & 7087.4 & 9308.77 & 91.01 & 91.37 \\
\hline 1971 & 228354.6 & 180939.3 & 4775.7 & 9412.9 & 9023.1 & 8736.88 & 85.11 & 89.91 \\
\hline 1972 & 257016 & 189464.3 & 5627.9 & 10421.3 & 11223.4 & 10372.24 & 101.23 & 90.46 \\
\hline 1973 & 326656.9 & 201772.4 & 5813.1 & 12356.5 & 15022.9 & 12475.78 & 120.38 & 93.20 \\
\hline 1974 & $358599 .<4$ & 213681.5 & 5626.2 & 12253.4 & 26899.4 & 15090.63 & 136.55 & 101.97 \\
\hline 1975 & 378983.3 & 222591.4 & 5844 & 11991.5 & 23959.6 & 15079.99 & 140.92 & 96.58 \\
\hline 1976 & 412968.4 & 227142.3 & 6172.7 & 12184.9 & 14154.2 & 13596.07 & 93.72 & 104.99 \\
\hline 1977 & 432214.1 & 234537.1 & 7126.2 & 13072 & 13430.2 & 11918.87 & 101.88 & 112.11 \\
\hline 1978 & 458268.3 & 251631.5 & 8918.7 & 14699.8 & 14225.7 & 14495.99 & 88.55 & 107.73 \\
\hline 1979 & 487599.9 & 271744.8 & 10255 & 16461.8 & 15627.9 & 19614.44 & 80.57 & 121.07 \\
\hline 1980 & 536051.8 & 306045.3 & 12885.4 & 20132 & 15510.1 & 26960.74 & 67.57 & 150.12 \\
\hline 1981 & 512469.2 & 332893.7 & 15980.3 & 25163.8 & 13455.3 & 31788.81 & 56.34 & 164.10 \\
\hline 1982 & 515575.5 & 330803.4 & 19420.8 & 23504.1 & 12255 & 18606.11 & 54.23 & 146.83 \\
\hline 1983 & 498003.7 & 316921.8 & 22467 & 21147.8 & 10150.4 & 11679.9 & 68.12 & 142.66 \\
\hline 1984 & 523205 & 328363.4 & 23724.7 & 25258.5 & 9766.8 & 14869.78 & 75.03 & 138.48 \\
\hline 1985 & 567169.7 & 336879.2 & 21543.7 & 25634 & 9611.7 & 17989.24 & 72.95 & 143.33 \\
\hline 1986 & 609646.8 & 323988.4 & 21955.9 & 22277.8 & 11725.4 & 15962.74 & 84.34 & 106.41 \\
\hline 1987 & 632019.8 & 328797.5 & 25520.5 & 26525.4 & 11535.5 & 16256.5 & 75.58 & 119.44 \\
\hline 1988 & 597795.1 & 307472.8 & 27104.7 & 31021.3 & 10845 & 23403.92 & 80.63 & 103.57 \\
\hline 1989 & 614789.9 & 320484 & 27557.7 & 39924.5 & 14279.1 & 30530.12 & 67.32 & 104.40 \\
\hline 1990 & 586004 & 337255.1 & 27956.7 & 37037.7 & 14684.4 & 35209.51 & 60.27 & 113.93 \\
\hline 1991 & 586620.2 & 351627 & 29956.7 & 36723.6 & 17389.3 & 41239.48 & 71.16 & 101.23 \\
\hline 1992 & 579999.7 & 364693.51 & 30828.2 & 42010.6 & 17888.6 & 51527.33 & 74.15 & 99.73 \\
\hline 1993 & 605797.7 & 371507.3 & 31710.1 & 47978.2 & 24469.1 & 50086.73 & 79.88 & 101.40 \\
\hline 1994 & 640184 & 388772.5 & 35287.3 & 48517.1 & 33475.5 & 58250.48 & 91.54 & 102.90 \\
\hline 1995 & 665422.2 & 363233.9 & 48438.3 & 46506.3 & 49857.5 & 46274.26 & 100 & 100.00 \\
\hline 1996 & 681909.6 & 383004 & 58727.1 & 46356.3 & 52241.2 & 58612.38 & 100.98 & 102.80 \\
\hline 1997 & 705579.9 & 409170.7 & 64941 & 50655.8 & 62674.2 & 58675.68 & 106.84 & 104.00 \\
\hline 1998 & 705140.4 & 429792.3 & 67493.4 & 51140 & 62759.8 & 76937.9 & 104.57 & 100.40 \\
\hline 1999 & 711832.4 & 445677.8 & 74827.9 & 48011 & 49272 & 85465.78 & 93.6 & 102.30 \\
\hline 2000 & 742164.6 & 475980 & 85204.8 & 55086 & 55783 & 81432 & 90.9 & 107.40 \\
\hline 2001 & 749505.7 & 474035.9 & 83485 & 70147.8 & 60744 & 93338.89 & 90.5 & 107.30 \\
\hline 2002 & 758852.6 & 478589.4 & 82865 & 76504.4 & 52641 & 112636.35 & 87.9 & 107.90 \\
\hline
\end{tabular}

